\title{
Cough Characteristics and FVC Maneuver in Cystic Fibrosis
}

\author{
Daphna Vilozni PhD, Moran Lavie MD, Miryam Ofek MD, Ifat Sarouk MD, and Ori Efrati MD
}

\begin{abstract}
BACKGROUND: Cough is part of life in patients with cystic fibrosis (CF). Weak coughing may add to increased respiratory disease that affects the mechanical properties of cough in these patients. In this study, we investigated cough characteristics in relation to forced expiratory flow/volume indices in CF. METHODS: This prospective study included 54 subjects $(26 \pm 10 \mathrm{y}$ old $)$ with CF. Similar indices of the maneuvers were compared. Additionally, other cough characteristics revealed by the maneuvers were investigated. Cough was considered efficient with 6 or more secondary spikes at a flow of $>2.67 \mathrm{~L} / \mathrm{s}$. RESULTS: Cough peak flows similar to peak expiratory flows (regardless of $\mathrm{FEV}_{1}$ severity level) were found in $98 \%$ of subjects. The secondary spike flows deteriorated alongside the percent-of-predicted $\mathrm{FEV}_{1}\left(\mathrm{r}^{2}=0.17, P=.002\right)$, yet inefficient secondary spike flows could also be found when $\mathrm{FEV}_{1}$ was within normal range. Mean efficient spike number was low $(2.5 \pm 1.2$ spikes/maneuver). Most cough spike flows were very small $(<0.9 \mathrm{~L} / \mathrm{s})$, resembling vibration that may contribute to the propulsion of mucus toward the central airways. Cough maneuver duration ended faster than forced expiration duration $(3.7 \pm 1.7 \mathrm{~s}$ vs $6.8 \pm 2.5 \mathrm{~s}, P<.001)$, resulting in a smaller cough vital capacity compared with expiratory vital capacity $(2.1 \pm 0.9 \mathrm{l}$ vs $3.1 \pm 0.7 \mathrm{l}$, $P<.001)$. Inspiratory volume below $2.23 \pm 0.07 \mathrm{~L}$ reduced efficient secondary spike number. CONCLUSIONS: The cough flow/volume maneuver reveals abnormalities in cough velocities and volume. A low secondary spike correlates with $\mathrm{FEV}_{1}$ severity level. The method may lead to earlier intervention to assist cough in CF. Key words: cystic fibrosis; spirometry; cough; lung mechanics. [Respir Care 2014;59(12):1912-1917. @ 2014 Daedalus Enterprises]
\end{abstract}

\section{Introduction}

Cough, the natural defense mechanism for airway clearance, is part of life for a patient with cystic fibrosis (CF), and frequent coughing is observed in $50 \%$ of infants with

\footnotetext{
The authors are affiliated with the Pediatric Pulmonary Unit and The National Center for Cystic Fibrosis, The Edmond and Lily Safra Children's Hospital, Sheba Medical Center, Ramat Gan, Israel, affiliated with the Sackler Faculty of Medicine, Tel Aviv University, Tel Aviv, Israel.

The study was supported by the J Baum Foundation of the Israel Lung Association, Tel Aviv, Israel.

The authors have disclosed no conflicts of interest.

Correspondence: Daphna Vilozni PhD, Pediatric Pulmonary Unit, The Edmond and Lily Safra Children's Hospital, Sheba Medical Center, Ramat Gan 52621, Israel. E-mail: daphna.vilozni@sheba.health.gov.il or avi_vil@bezeqint.net.
}

DOI: $10.4187 /$ respcare. 03290
CF by the age of 10 months. ${ }^{1,2}$ Weak coughing is perceived as an additional cause of recurrent respiratory infections, leading to respiratory disease characterized by deterioration of lung function in CF. ${ }^{1-4}$

The sequence of events that lead to an effective cough, which has long been known, consists of inspiratory, compressive, and expiratory phases. ${ }^{5-8}$ The initial phase of cough is characterized by inhalation of gas that may be as little as $50 \%$ of the tidal volume or as great as $50 \%$ of the vital capacity (VC). ${ }^{9}$ However, inhalation of a large volume of gas will produce greater lengthening of the expiratory muscles, which will generate greater positive intrathoracic pressures for a given expiratory phase. ${ }^{9}$

The second phase of cough depends on expiratory muscle strength and adequate control of the upper airway to provide an explosive release of peak expiratory flow (PEF). ${ }^{5-8}$ The commonly used parameters to assess this phase are the cough peak flow $(\mathrm{CPF})$ and $\mathrm{CPF} / \mathrm{PEF}$ ratio. ${ }^{10}$ These parameters were found to be normal in most patients with $\mathrm{CF}$ and to correlate with patient body mass index (BMI) level. ${ }^{11}$ 


\section{Cough and FVC Maneuver In CF}

The third phase of cough is continuation of exhalation, where the bronchi and trachea are compressed and narrowed in the intrathoracic space during coughing (or forced expiration). In healthy subjects, the narrowing is evenly distributed, and the expiratory decay is smooth. In patients with airway obstruction such as in $\mathrm{CF}$, the distribution of narrowing may be greater, more irregular, and sometimes the cause of near collapse of the main bronchi. .-8 $^{-8}$ Moreover, the high pressures developed during the expiratory explosion may cause airway collapse in the presence of airway obstruction and changes in airway structure. ${ }^{8}$

The expiratory decay of the cough flow consists of transient supramaximal flow (higher than PEF) cough spikes. The number of spikes/cough epoch ranges from 8 to 14 in a healthy adult. ${ }^{5-8}$ Cough spike flows between 160 and $180 \mathrm{~L} / \mathrm{min}(2.67-3.0 \mathrm{~L} / \mathrm{s})$ were found to provide effective airway clearance in adult patients with stable neuromuscular disease who still had bulbar function. ${ }^{12}$

The forced expiratory and cough flow/volume maneuvers link in several indices. These include the inspiratory capacity, peak flows, and VC. We hypothesized that although CPFs may be normal, early airway collapsibility during voluntary cough maneuvers may pathologically affect secondary cough spike flows in these patients.

The aim of this study was to investigate the similarity and differences between the cough and forced expiratory flow/volume maneuver indices. Additionally, we explored the cough flow/volume index characteristics in relation to actual anthropomorphic data retrieved from subjects with normal lung function and from subjects with bacterial infections. Indices were also related to the retrospective FEV1 yearly deteriotation rate. ${ }^{13}$

\section{Methods}

\section{Subjects}

Patients with CF who were treated at The National Center for Cystic Fibrosis at The Edmond and Lily Safra Children's Hospital (Ramat Gan, Israel) were enrolled in this study. For inclusion, subjects had to be older than $10 \mathrm{y}$ and to be able to comply with both the FVC and cough flow/volume maneuvers. Subjects were excluded if they had respiratory exacerbations, or were hospitalized for other reasons.

Clinical charts were reviewed for the following data: age, BMI, age and gender percentiles, and genetics.

\section{Study Design}

This was a prospective and cross-sectional study. It was approved by the Sheba Medical Center Helsinki Review Board (IRB 8709-11-SMC, NCT01636219). Measurements were performed after each subject signed an

\section{QUICK LOOK}

\section{Current knowledge}

Cough is both a symptom and protective mechanism in cystic fibrosis (CF). Weak or ineffective cough results in secretion retention, infection, and deterioration of lung function. Cough effectiveness is linked to the degree of $\mathrm{FEV}_{1}$ impairment.

\section{What this paper contributes to our knowledge}

The measurement of the cough flow/volume maneuver in $\mathrm{CF}$ demonstrates abnormalities in cough velocity and volume. A low secondary spike in the maneuver correlates with the severity of $\mathrm{FEV}_{1}$ impairment and can identify patients for early intervention to improve cough and secretion clearance.

informed consent form. Tests were performed before physiotherapy, and no inhaled medications (such as bronchodilators, hypertonic saline, and antibiotic inhalations) were given $12 \mathrm{~h}$ before the tests.

Measurements. Both forced expiratory flow/volume and cough flow/volume maneuvers were measured with the KoKo spirometer (nSpire Health, Longmont, Colorado): data sampling rate, 200 samples/s; flow range, $\pm 16 \mathrm{~L}$; volume range, $0-19.9 \mathrm{~L}$; and accuracy, $\pm 2 \%$. Measurements were performed while the subject was standing to standardize both measurements because the cough maneuver can be performed better while standing.

Forced Flow/Volume Maneuver Test. The forced expiratory maneuvers were performed according to guidelines for quality control. ${ }^{14,15}$ During the test, the subject was asked to wear a nose clip, and the mouth was sealed around the mouthpiece. The subject was then asked to take a full inspiration from residual volume to total lung capacity, considered inspiratory $\mathrm{VC}$, and blow into a pneumotachograph. At least 3 maneuvers were performed. The best curve $\left(\mathrm{FEV}_{1}+\mathrm{FVC}\right)$ was stored for further analysis.

Cough Flow/Volume Maneuver Tests. Tests were performed $\sim 5$ min after spirometry. ${ }^{16}$ While wearing a nose clip and with the mouth sealed around a mouthpiece, the subject took a full inspiration from residual volume to total lung capacity (cough inspiratory VC) and coughed as forcefully as possible through the sealed mouthpiece into the pneumotachograph without intervening inspirations between the coughing spike effort. Up to 3 cough maneuvers were performed. The cough maneuver chosen for analysis was the one with the highest cough inspiratory $\mathrm{VC}$ and the best CPF. Other cough flow/volume parame- 


\section{Cough And FVC Maneuver IN CF}
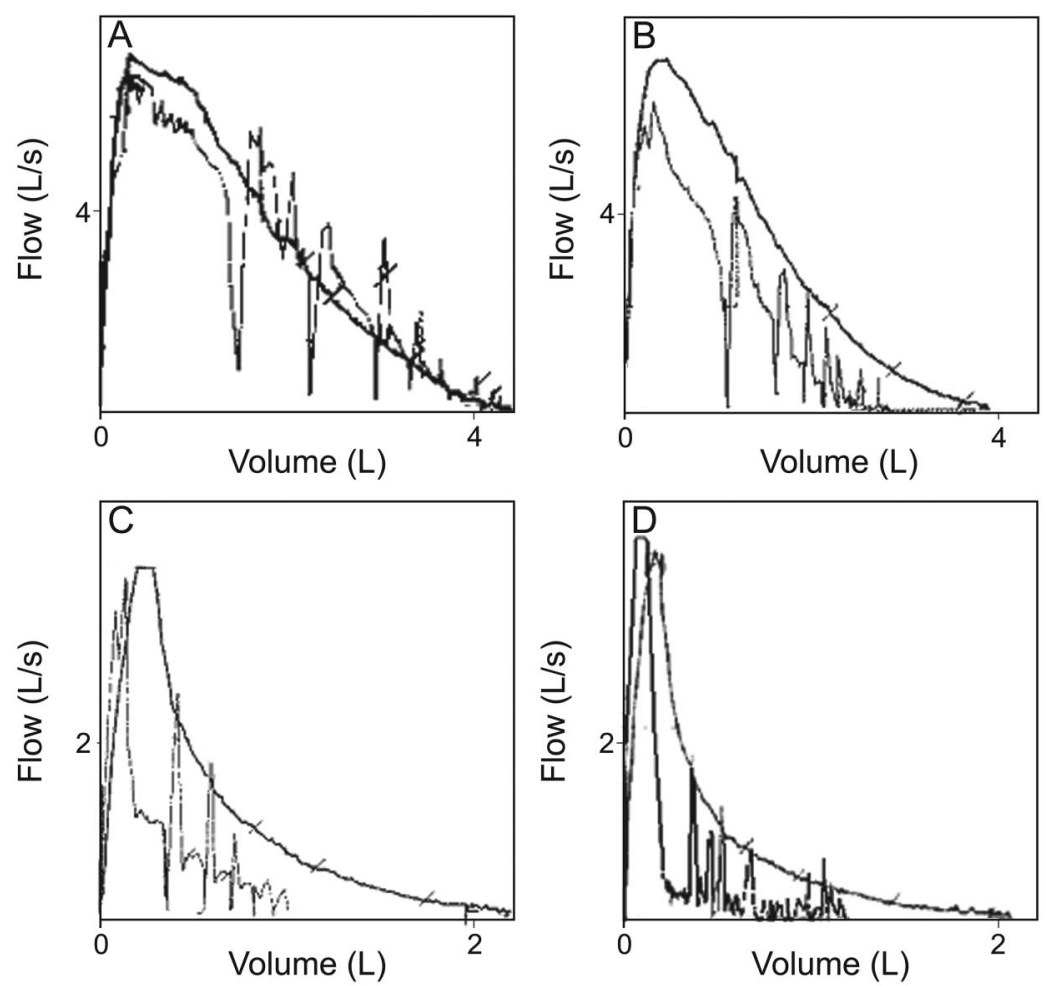

Fig. 1. Representative scanned forced flow/volume curves and cough flow/volume curves. The cough flow/volume curve presented has several spikes. A: $\mathrm{FEV}_{1}=95 \%$ of predicted; B: $\mathrm{FEV}_{1}=73 \%$ of predicted; C: $\mathrm{FEV}_{1}=40 \%$ of predicted; D: FEV $1=25 \%$ of predicted.

ters were defined similarly to the conventional expiratory flow/volume indices.

\section{Data Analysis}

Analysis of the cough maneuver included the cough inspiratory capacity volume, CPF, cough spike flows, and the number of spikes. An efficient cough was defined as having at least 6 spikes at a flow of $>160 \mathrm{~L} / \mathrm{min}$ $(2.67 \mathrm{~L} / \mathrm{s}) .{ }^{12}$ Analysis of the flow/volume indices included inspiratory capacity, PEF, FVC, and $\mathrm{FEV}_{1}$. Reference values of the spirometry $\left(\mathrm{FEV}_{1}\right)^{17}$ were also used to predict cough inspiratory VC and cough VC. Correlations were sought between severity of airway disease classified by the percent-of-predicted $\mathrm{FEV}_{1}$ and other cough mechanical properties (number of spikes, spike flows, and cough inspiratory VC). In addition, we sought to determine whether the $\mathrm{FEV}_{1}$ yearly deterioration rate ${ }^{13}$ affects some cough characteristics.

\section{Statistics}

We used the Prism statistical software package (GraphPad Software, San Diego, California). The data were tested for normal distribution by the Kolmogorov-Smirnov test. Normally distributed variables were reported as mean $\pm \mathrm{SD}$ and range. When data had no normal distribution, variables were also reported as median and range and were analyzed using the paired-sample $t$ test or the Wilcoxon signed-rank test. Significant differences between cough flow/volume maneuver indices and FVC indices were compared by paired $t$ tests (Mann-Whitney tests). Correlations between continuous variables were assessed using Pearson correlation coefficient tests when variables were normally distributed or the Spearman test. Correlations between cough parameters were also studied in relation to BMI and other CF comorbidities and in relation to yearly deterioration in $\mathrm{FEV}_{1} \cdot{ }^{13} P<.05$ was considered significant.

\section{Results}

Fifty-four subjects with CF (31 males) participated in this study. The mean age was $26 \pm 10$ y (median age of $26 \mathrm{y}$ with $95 \%$ confidence limit, range of 23-29 y). The mean BMI was $26 \pm 23 \mathrm{~kg} / \mathrm{m}^{2}$. Mutation severity distribution included 33 subjects with severe mutation (classes I-III) and 21 subjects with milder mutation (classes IV and V). Twenty-six patients (48\%) were infected with Pseudomonas.

Several typical superimposed curves of forced expiratory and cough maneuvers are presented in Figure 1. The 
Table 1. Measured Values of Cough and Forced Expiratory Indices

\begin{tabular}{|c|c|c|c|c|c|}
\hline Parameter & & & $\begin{array}{l}\text { Cough } \\
\text { Maneuver }\end{array}$ & $\begin{array}{l}\text { Expiratory } \\
\text { Maneuver }\end{array}$ & $P$ \\
\hline $\begin{array}{l}\text { Inspiratory vital } \\
\text { capacity, L }\end{array}$ & & & $3.0 \pm 0.8$ & $3.1 \pm 0.9$ & .54 \\
\hline FVC, L & & & $2.1 \pm 0.9$ & $3.1 \pm 0.7$ & $<.001$ \\
\hline $\begin{array}{l}\text { Expiratory } \\
\text { duration, s }\end{array}$ & & & $3.7 \pm 1.7$ & $6.8 \pm 2.5$ & $<.001$ \\
\hline Peak flows, L/s & & & $6.0 \pm 2.0$ & $5.9 \pm 2.0$ & .80 \\
\hline \multirow[t]{3}{*}{$\mathrm{CPF} / \mathrm{PEF}$ ratio } & & & $1.0 \pm 0.2$ & & \\
\hline & \multicolumn{2}{|c|}{ Subjects } & & & \\
\hline & $n$ & $\%$ & & & \\
\hline Spike 2, L/s & 53 & 98 & $3.6 \pm 1.5$ & & \\
\hline Spike 3, L/s & 53 & 98 & $2.6 \pm 1.3$ & & \\
\hline Spike $4, \mathrm{~L} / \mathrm{s}$ & 45 & 83 & $1.6 \pm 1.2$ & & \\
\hline Spike $5, \mathrm{~L} / \mathrm{s}$ & 31 & 57 & $0.9 \pm 1.1$ & & \\
\hline Spike 6, L/s & 16 & 30 & $0.4 \pm 0.7$ & & \\
\hline
\end{tabular}

$n=$ number of subjects who performed efficient spikes (if $<54$ subjects).

$\mathrm{CPF}=$ cough peak flow

$\mathrm{PEF}=$ peak expiratory flow

cough flow/volume curve contains several spikes. Figure 1 shows that the peak flows of cough and forced expiration were similar, regardless of $\mathrm{FEV}_{1}$ severity. However, the flow decay of the curves differs. Cough flow decay was faster compared with expiratory flow decay in relation to $\mathrm{FEV}_{1}$ severity. Cough spikes with very small flow amplitudes $(>0.9 \mathrm{~L} / \mathrm{s})$ resembling vibration also increased with $\mathrm{FEV}_{1}$ deterioration.

The actual measured values for the cough and forced expiratory flow/volume maneuvers for the entire group are presented in Table 1. The median $\mathrm{FEV}_{1}$ was $65.8 \%$ of predicted (range of 19.8-116.1). Similarities between maneuvers were limited to inspiratory capacity before CPF and to the peak flows. The ratio between the peak flows was normal. The cough VC significantly differed from the FVC. A cough should include 6 spikes; however, not all subjects could produce all 6 spikes. All subjects could perform one spike; but there was a decline in the number of subjects able to perform 2,3, or more spikes. Coughing bout duration was significantly shorter than expiration time. Cough maneuver duration was shorter than forced expiration time, resulting in a smaller cough VC compared with expiratory VC.

The effect of inspiratory cough capacity values on the number of efficient spikes is presented in Figure 2. Third and fourth efficient secondary spikes were initiated mostly when inspiratory capacity was $>2.23 \pm 0.07 \mathrm{~L}$.

The effect of percent-of-predicted $\mathrm{FEV}_{1}$ on $\mathrm{CPF}$ and secondary spike flows is presented in Figure 3. There was no correlation between percent-of-predicted $\mathrm{FEV}_{1}$ and $\mathrm{CPF}$ or secondary cough spike flow. CPF was reduced in

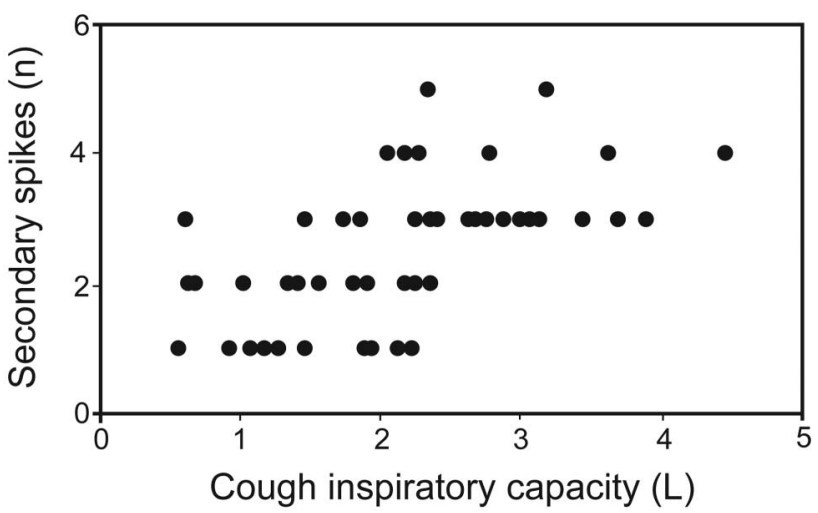

Fig. 2. Effect of inspiratory cough capacity values on the number of efficient spikes $\left(r^{2}=0.35, P<.001\right)$. Third and fourth sufficient secondary spikes were initiated mostly when inspiratory capacity prior to CPF was above $2.23 \pm 0.07 \mathrm{~L}$.
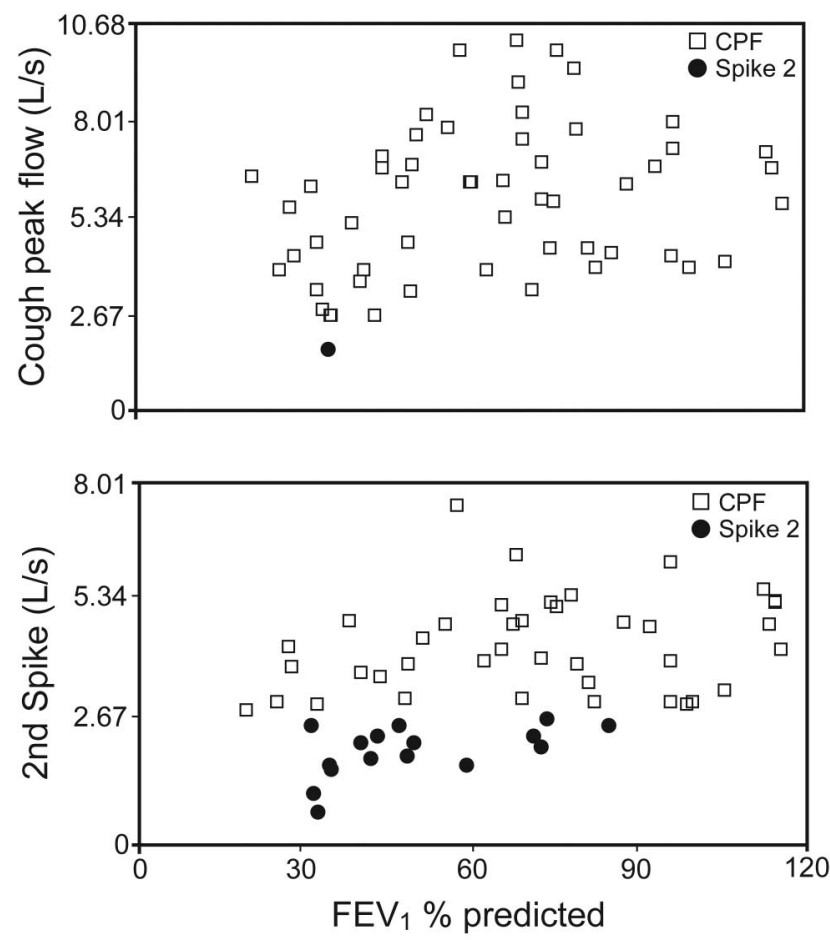

Fig. 3. Effect of percent-of-predicted $\mathrm{FEV}_{1}$ on cough peak flow (CPF) and secondary spike flow. Correlations did not reach significance, yet CPFs were reduced in a single subject who had an $\mathrm{FEV}_{1}$ of $35 \%$ of predicted, whereas secondary spike flows were reduced across percent-of-predicted $\mathrm{FEV}_{1}$.

a single subject who had an $\mathrm{FEV}_{1}$ of $35 \%$ of predicted. Secondary spike flow values were reduced across the percent-of-predicted $\mathrm{FEV}_{1}$.

The effect of the yearly deterioration rate for $\mathrm{FEV}_{1}$ and CPF or secondary spike flows is presented in Figure 4. Both CPF and secondary spike flows significantly correlated with $\mathrm{FEV}_{1}$ yearly deterioration rate $\left(\mathrm{r}^{2}=0.33\right.$, $P<.001$; and $\mathrm{r}^{2}=0.36, P<.001$, respectively). How- 


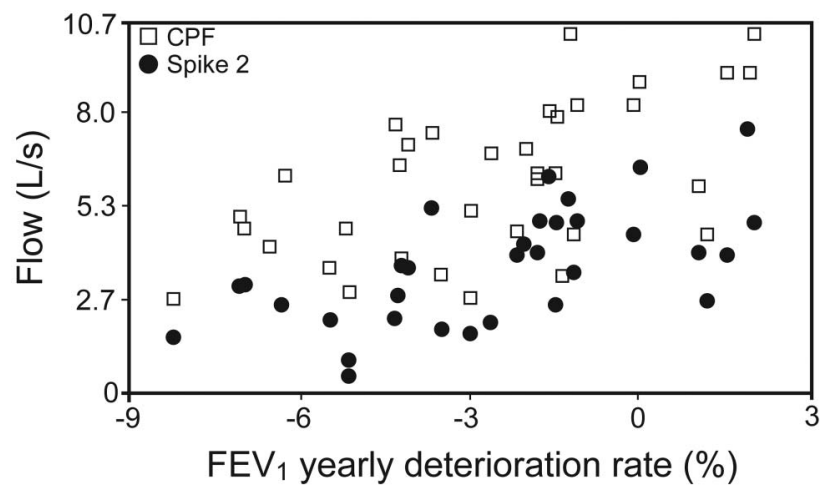

Fig. 4. Effect of the yearly deterioration rate in $\mathrm{FEV}_{1}$ and cough peak flow (CPF) or secondary spike flows $\left(r^{2}=0.32, P=.002\right)$.

ever, the actual CPF values were not reduced below efficiency, whereas the secondary spike flow values deteriorated below efficiency along with the $\mathrm{FEV}_{1}$ yearly deterioration rate. Subjects with $\mathrm{FEV}_{1}$ yearly deterioration rates of $3.0 \%$ and higher tended to have low secondary spike flows.

We found a positive correlation between BMI (percentile) and secondary spike flow $\left(\mathrm{r}^{2}=0.12, P=.01\right)$. When the BMI was $<20$ th percentile, $\mathrm{CPF}$ was reduced in 2 subjects, and secondary spike flows were reduced in 15 subjects. Three subjects with BMI in the 50th, 65th, and 85th percentiles also had reduced secondary spike flows. We did not find a correlation between secondary spike flow efficiency and mutation severity. We could not establish a correlation between secondary spike flow values and Pseudomonas infection. Seven of 28 subjects without Pseudomonas and 10 of 26 subjects with Pseudomonas had low secondary spike flows, but the differences did not reach significance.

\section{Discussion}

In this study, we investigated cough characteristics in relation to lung function in subjects with $\mathrm{CF}$. The findings indicate that although cough inspiratory $\mathrm{VC}, \mathrm{CPF}$, and $\mathrm{CPF} / \mathrm{PEF}$ ratios were relatively preserved in our subjects, other tested parameters were obstructed. These included decrease in secondary spike flows, limited number of efficient spikes/cough maneuver, and short duration of each coughing bout leading to reduced cough VC. These findings correlated with the decrease in percent-ofpredicted $\mathrm{FEV}_{1}, \mathrm{FEV}_{1}$ yearly deterioration rate, and BMI (percentile). However, the impaired parameters could also be observed when the percent-of-predicted $\mathrm{FEV}_{1}$ and BMI were within the normal range.

Our findings that CPF is efficient in CF are strengthened by former studies. All but one subject could produce $\mathrm{CPF}$ values above the lower limit for efficient cough ca- pacity. Indeed, $\mathrm{CPF}$ and $\mathrm{CPF} / \mathrm{PEF}$ values were within the expected range to produce an efficient cough. Apparently, these values were preserved despite deterioration in percent-of-predicted $\mathrm{FEV}_{1}$ and despite inefficiently low inspiratory VC. The findings imply that the expiratory muscles are well preserved. Dassios et al ${ }^{9,18}$ likewise found that subjects with $\mathrm{CF}$ have adequate maximal expiratory pressures, $\mathrm{CPF}$, and $\mathrm{CPF} / \mathrm{PEF}$ ratios, and they suggested that the expiratory pressures needed for CPFs are preserved in patients with $\mathrm{CF}$.

We also found that the cough inspiratory $\mathrm{VC}$ volume did not limit the CPF values, as found in a former study, ${ }^{10}$ but the amount of air (L) inhaled before the cough (cough inspiratory VC) did affect the number of efficient secondary spikes (see Fig. 2). This finding may be related to the volume used during the $\mathrm{CPF}$, but we could not measure that volume. Chaudri et al ${ }^{19}$ studied the secondary spike efficiency in motor neuron diseases and found an association between the absence of efficient cough spikes and increased mortality.

Our findings suggest that secondary spike flows elucidate cough deterioration better than the $\mathrm{CPF}$ or $\mathrm{CPF} / \mathrm{PEF}$ ratio. Indeed, secondary spike flow values correlated with percent-of predicted $\mathrm{FEV}_{1}$ deterioration (see Fig. 3), but inefficient low values were obtained even when $\mathrm{FEV}_{1}$ was normal. Interestingly, we also found that most subjects with a yearly deterioration rate higher than $3 \%$ also had low secondary spike flows (see Fig. 4). The dependent variable here has yet to be discovered.

Furthermore, we found that secondary spike flows were inefficient when BMI was $\leq 18 \mathrm{~kg} / \mathrm{m}^{2}$ (20th percentile), but low secondary spike flows were likewise seen even when BMI was normal. The connection between low BMI and lung function deterioration has been established. ${ }^{11}$ The secondary cough spike characteristics in relation to $\mathrm{FEV}_{1}$ reinforce the need to study not only CPF but also other cough parameters.

The decay of cough spike flows was parallel to the decay of the forced expiratory flow maneuver only at the beginning of expiration, which included the portion between the PEF and forced expiratory flow at $25 \%$ of $\mathrm{VC}$. After that, the spike flows rapidly decreased (see Fig. 1) compared with the expiratory maneuver, whereas the cough epoch duration was shorter compared with the forced expiratory duration. We therefore attribute the short cough time to a smaller cough FVC volume compared with FVC (see Table 1), as found in our study and corroborated previously. ${ }^{5-8}$ The pathophysiology underlying our findings may be explained by the high collapsibility of airways, diffuse and severe inflammation, thickening of airway walls, presence of bronchiectasis, and abnormal quantity or quality of mucus lodged in airways, which are all found in CF patients with moderate-to-severe airway disease. ${ }^{20,21}$ 


\section{Cough and FVC Maneuver In CF}

Rapid very small spikes with flows below $0.9 \mathrm{~L} / \mathrm{s}$ (seemingly vibration-like amplitudes) were apparent from midexpiratory flows to the end of the cough maneuver $\left(\mathrm{FEV}_{1}\right.$ is below $40 \%$ predicted values; see Fig. 1). We suggest that these rapid very small flow amplitudes contribute to the propulsion of small amounts of liquids and mucus toward the central airways. As mucus clearance is directly proportional to mucus rheology (it is inversely proportional to its viscosity and elasticity depending on the depth at which it is situated in the airways), ${ }^{20}$ it is not surprising that patients with $\mathrm{CF}$ may require up to 14-39 cough epochs/h to overcome the sticky mucus in their airways. ${ }^{21,22}$

The main limitation of this study is that the cough flow/volume maneuver is not yet standardized, and no reference values exist for secondary spike values in the healthy population. Therefore, we had to refer to older publications for values for healthy subjects. However, our findings revealed abnormalities in cough velocity that were connected with the descending portion of the flow/volume curve and are evident in CPF alone.

The cough flow/volume maneuver is always measured after spirometry, and we feel that it would have been better to use a random order. However, the cough flow/volume maneuver is a fatiguing process, and we therefore decided on this fixed order. One could argue that the findings are related to fatigue due to the repetition of spirometry tests before the cough maneuver. We propose that fatigue will manifest as poor CPF, but the expiratory portion will not be influenced by fatigue.

We conclude that the cough flow/volume maneuver used in our study reveals abnormalities in cough characteristics that may not be evident with the CPF method alone. All other parameters (secondary spike flows, number of secondary spikes, cough duration, and cough VC) were found to be low in many of our subjects with $\mathrm{CF}$. Inefficient secondary spikes, in particular, were found to be directly correlated with percent-of-predicted $\mathrm{FEV}_{1}$, $\mathrm{FEV}_{1}$ deterioration rate, and low BMI, but these were also apparent even when $\mathrm{FEV}_{1}$ or BMI was normal. The use of the cough flow/volume maneuver with the measurement may therefore be justified and may be clinically important in $\mathrm{CF}$, but further standardization is required to recommend its use.

\section{REFERENCES}

1. Farrell PM, Li Z, Kosorok MR, Laxova A, Green CG, Collins J, et al. Longitudinal evaluation of bronchopulmonary disease in children with cystic fibrosis. Pediatr Pulmonol 2003;36(3):230-240.

2. Mott LS, Park J, Murray CP, Gangell CL, de Klerk NH, Robinson $\mathrm{PJ}$, et al. Progression of early structural lung disease in young children with cystic fibrosis assessed using CT. Thorax 2012;67(6): 509-516.
3. Ratjen F, Döring G. Clinical manifestations of pulmonary disease in cystic fibrosis. Lancet 2003;361(9358):681-689.

4. Rosenfeld M, Emerson J, Williams-Warren J, Pepe M, Smith A, Montgomery AB, Ramsey B. Defining a pulmonary exacerbation in cystic fibrosis. J Pediatr 2001;139(3):359-365.

5. Knudson RJ, Mead J, Knudson DE. Contribution of airway collapse to supra-maximal expiratory flows. J Appl Physiol 1974;36(6): 653-667.

6. Pedersen OF, Lyager S, Ingram RH Jr. Airway dynamics in transition between peak and maximal expiratory flow. J Appl Physiol 1985;59(6):1733-1746.

7. Naire S. Dynamics of voluntary cough maneuvers: a theoretical model. J Biomech Eng 2009;131(1):011010.

8. McCool FD. Global physiology and pathophysiology of cough: ACCP evidence-based clinical practice guidelines. Chest 2006;129(1 Suppl): 48S-53S.

9. Dassios TG, Doudounakis S, Dimitriou G. Maximum rate of pressure development and maximal relaxation rate of respiratory muscles in patients with cystic fibrosis. Respir Care 2013;58(3):474-481.

10. Smith JA, Aliverti A, Quaranta M, McGuinness K, Kelsall A, Earis J, Calverley PM. Chest wall dynamics during voluntary and induced cough in healthy volunteers. J Physiol 2012;590(Pt 3):563-574.

11. Steinkamp G, Wiedemann B. Relationship between nutritional status and lung function in cystic fibrosis: cross sectional and longitudinal analyses from the German CF quality assurance (CFQA) project. Thorax 2002;57(7):596-601.

12. Bach JR, Saporito LR. Criteria for extubation and tracheostomy tube removal for patients with ventilatory failure. A different approach to weaning. Chest 1996;110(6):1566-1571

13. Vilozni D, Lavie M, Sarouk I, Efrati O. Progressive flow-to-volume dysanapsis in cystic fibrosis: a predictor for lung transplantation? Am J Respir Crit Care Med 2012;186(1):82-87.

14. Miller MR, Hankinson J, Brusasco V, Burgos F, Casaburi R, Coates A, et al. Standardization of spirometry. Eur Respir J 2005;26(2): 319-338.

15. Beydon N, Davis SD, Lombardi E, Allen JL, Arets HG, Aurora P, et al. An official American Thoracic Society/European Respiratory Society statement: pulmonary function testing in preschool children. Am J Respir Crit Care Med 2007;175(12):1304-1345.

16. Beardsmore CS, Wimpress SP, Thomson AH, Patel HR, Goodenough $\mathrm{P}$, Simpson H. Maximum voluntary cough: an indication of airway function. Bull Eur Physiopathol Respir 1987;23(5):465-472.

17. Quanjer PH, Stanojevic S, Cole TJ, Baur X, Hall GL, Culver BH, et al. ERS Global Lung Function Initiative. Multi-ethnic reference values for spirometry for the 3-95-yr age range: the global lung function 2012 equations. Eur Respir J 2012;40(6):1324-1343.

18. Dassios T, Katelari A, Doudounakis S, Mantagos S, Dimitriou G. Respiratory muscle function in patients with cystic fibrosis. Pediatr Pulmonol 2013;48(9):865-873

19. Chaudri MB, Liu C, Hubbard R, Jefferson D, Kinnear WJ. Relationship between supramaximal flow during cough and mortality in motor neurone disease. Eur Respir J 2002;19(3):434-438.

20. King M. The role of mucus viscoelasticity in cough clearance. Biorheology 1987;24(6):589-597.

21. Hamutcu R, Francis J, Karakoc F, Bush A. Objective monitoring of cough in children with cystic fibrosis. Pediatr Pulmonol 2002;34(5): 331-335.

22. Smith JA, Owen EC, Jones AM, Dodd ME, Webb AK, Woodcock A. Objective measurement of cough during pulmonary exacerbations in adults with cystic fibrosis. Thorax 2006;61(5):425-429. 